\title{
Towards an Introduction to Computational Semiotics
}

\author{
Ricardo Gudwin \& João Queiroz \\ DCA-FEEC-UNICAMP, Av. Albert Einstein 400, \\ 13083-852 Campinas, SP - Brazil \\ gudwin@dca. fee. unicamp.br \\ http://www.dca.fee.unicamp.br/ gudwin \\ queirozj@dca.fee.unicamp.br \\ http://www.digitalpeirce.org/joao
}

\begin{abstract}
Computational Semiotics is a quite new field of research, which emerged from advanced studies on artificial intelligence and intelligent systems. It is an alternative to the mainstream approaches - those based either on cognitivism or on biologically inspired techniques. Instead, Computational Semiotics seeks its inspiration on semiotics, a tradition on the philosophy of mind dealing with the concepts of representation and communication from a more technical perspective. The whole story besides this new field of research is usually unknown from many researchers working on the field of intelligent systems. This story started during the 1970's, where two main precursors first published works relating semiotics to intelligent systems development, almost at the same time in Russia and in the U.S. These pioneering studies were performed by Dmitri Pospelov in Russia and Eugene Pendergraft in U.S. Despite promising research results, for diverse reasons they stayed ignored from the mainstream in Artificial Intelligence for about 25 years. However, around 1995 they were rediscovered, motivating the development of this new paradigm of research. The goal of this paper is to briefly review the main contributions in this area.
\end{abstract}

Keyword:- Computational Semiotics, Intelligent Systems, Artificial Intelligence, Artificial Cognition.

\section{INTRODUCTION}

Semiotics is a field of research involved in the study of meaning and communication processes (semiosis), from the point of view of formal sciences, linguistics and philosophy [1]. Systematic attempts of interdisciplinary studies involving semiotics and intelligent systems were independently developed by researchers from Russia and U.S., during the 60 's and 70's. The first russian proposal appeared on the work of Dmitri Pospelov [2,3], whose original coverage of intelligent control theory is still widely unknown on the western science. On the U.S. a similar effort appeared on the work of Eugene Pendergraft [4,5], also somewhat unknown to the mainstream. Despite being ignored for about 25 years, a new growing interest for such works started to appear in conferences around $1995^{1}$ and have been increasing since then. Many research lines were originated during these meetings. The main purpose of this paper is to present a brief overview of the most representative works in the field, referring the reader to the relevant literature required to those interested in a deeper understanding of those issues. In the following sections, we present a walk-through on the many approaches on the field, focusing on the distinctive characteristics of each approach.

\section{Semiotic Situational Control}

Parallel to the history of artificial intelligence in the western world, a sequence of developments was undertaken in Russia, with similar efforts and ideas. At the end of the 60ths and beginning of 70ths, while mathematical logic, situational calculus and knowledge-based systems started to appear in the West, situational control, an expert-systemslike kind of technology started in Russia through the work of Pospelov [2,6]. Situational control can be viewed as a russian version of situational calculus applied to the automatic control of systems. Going beyond western situational calculus, though, Pospelov, and followers [3,7,8,9], further created a development of situational control which they called semiotic situational control (SSC) [3, pp. 322]. This

${ }^{1}$ Workshop on Architectures for Semiotic Modeling and Situation Analysis in Large Complex Systems, $10^{\circ}$ IEEE International Symposium on Intelligent Control, Monterey, CA, 1995; Workshop on Control Mechanisms for Complex Systems: Issues of Measurement and Semiotic Analysis, Las Cruces, New Mexico, USA, 8-12 december 1996; Second Workshop on Applied Semiotics, Smolenice Castle Slovakia, 15 september 1997; ISAS'97 - Intelligent Systems and Semiotics - A Learning Perspective - International Conference - Gaithersburg, USA, 22-25 september 1997; ISIC/CIRA/ISAS'98 - IEEE International Symposium on Intelligenct Control/ Computational Intelligence in Robotics and Automation/ Intelligent Systems and Semiotics - Gaithersburg, USA, 14-18 september 1998; ISIC/ISAS'99 - IEEE International Symposium on Intelligent Control, Intelligent Systems and Semiotics - Cambridge, MA - USA, 15-17 September 1999; the International Conference on Intelligent Systems and Semiotics (ISAS) - which from 1996 to 1998, happened in Gaithersburg, Maryland, USA, being organized and sponsored by NIST (National Institute on Standards in Technology), and also sponsored by IEEE. 
term was first employed in a seminar organized in Moscow in 1971 with the tittle "Semiotic Methods of Control in Large Systems" [3, pp. 322]. Pospelov's interest was on "open large complex systems" (ecological and social systems, large comercial or industrial corporations, political parties), systems unsuitable to be controlled by conventional control techniques. Pospelov's goal was to emulate artificially the abitity of human beings in controlling such kind of systems, following the same motivation that turned into the creation of knowledge-based systems in the West. The guidelines used in situational control were somewhat equivalent to those used in knowledge-based systems: the possible states of the system under control were characterized in terms of the description of situations suitable to happen; these situations were then classified in terms of more general and abstract situations; and then logiclinguistic rules were used to describe the changes from situation to situation [3, pp. 322] on the states of the system. The main issue on semiotic situational control was then to use linguistic propositions regarding situations and linguistic rules to describe the dynamics of system and its control. Nothing necessarily new, when compared to western similar approaches. But Pospelov's SSC was more than its knowledge-based counterpart, due to the kind of systems under perspective. In West, rule-based control systems were used mainly to control non-linear physical systems, industrial and/or manufacturing processes. In Russia, the complexity of the systems under investigation - open large complex systems, required further developments for SSC. Differently from logic-linguistic models developed on the West, terms and rules were not just ungrounded symbols building purely syntactical systems. The formalization of SSCs took into account sophistications like the grounding of linguistic terms and rules (its semantics), the existence of rules to govern the change into terms and rules semantics, and a hierarchical description of the system in terms of many different levels of resolution.

A semiotic situational control system (SSCS) is composed by a set of different control modules, organized into a multilevel (hierarchical) structure for the control of open large complex systems. It is characterized by the use of many different modeling artifacts and tools, depending on the module and its level on this structure: (i) A Semiotic Model (SM) which is a kind of a general metamodel, governing the structure and behavior of different parts of this structure; (ii) A Discrete Situational Network (DSN) which describes the system under control as a discrete event dynamical system; (iii) A Situational Control Language (SCL) which allow the description of knowledge about the system, with its semantics mapped on the states of the DSN; (iv) A Semiotic Knowledge Base (SKB) which stores sentences in SCL; (v) A Semiotic Solver (SS) which processes the knowledge in SKB generating control decisions.

The Semiotic Model is a mathematical extension for the notion of a formal model. The basic assumption is that in a formal model, each term has a fixed and unique meaning, and in a semiotic model, this meaning can be changed as the system works. Semiotic models are used throughout a SSCS to provide its mathematical consistency and strictness. The Discrete Situational Network is the first level of modeling for the system under control. Under this level, the controlled system is viewed as a discrete event dynamical system, encoded by means of a network of automata. Each system component is described as an automata, with inputs and outputs. Outputs from one automata are directed to inputs of other automata connected to it by the network. Inputs cause the change of its internal state, and this change will also cause an output, which is conveyed to the next automata and so on. Situations on the DSN are described by means of expressions in a Situational Control Language. A Semiotic Knowledge Base, containing a list of general situations and possible transitions between situations, expressed in SCL, stores the general knowledge assumed for the system under control. Finaly, a Semiotic Solver uses the information extracted from the DSN (and interpreted by means of expressions on SCL) to generate the control decisions.

As we stated earlier, one important difference of the SSCS when compared to a knowledge-based system is the grounding of terms and rules. In knowledge based systems, the meaning of sentences in a knowledge base (their ground on the real wordl) is given by a human person, the knowledge engineer [10], which encodes knowledge about the real world into symbols to be processed by the control system. At the SSCS the meaning of sentences is grounded not on the real world, but on the states of a DSN. Differently from a knowledge based system, the semantic of expressions in SCL have a very precise meaning, based solely on the DSN, and independent from an external human interpretation. By attributing the semantics of expressions to DSN states, it is possible to determine a language with a very well defined semantics (see [3], pps. 55-67 for examples). The SCL presents structures similar to a natural language, including concepts, names, relations, actions, evaluations, quantifiers and modal operators, which are mapped onto the elements of a DSN. These structures are used to describe the discrete situations found on the network, and also for the behavior of the system.

SSCSs were used successfully in many different kinds of applications, e.g. car traffic control, cargo handling operations in sea ports, dispatching and operational control of technical organizations [3]. The work of Pospelov opened a whole subfield of investigation, both in Russia and in the U.S., which is referred today in the literature as Applied Semiotics [8]. It is interesting to notice three important contributions on the work of Pospelov, which grants him a special position as a precursor on the history of computational semiotics. The first one is its idea of grounding symbols of a knowledge-based language onto states of a discrete-event tool - the DSN, instead of directly on the real world. The second one is related to the way he focused learning in his system, conceptualized as a fundamental part of the semiotic model - no symbol has a fixed meaning, but 
a meaning that can always change if necessary. This allows the system to grow its repertoire of symbols and tune their meanings by interacting with the environment. As soon as a new information is introduced on the system, which cannot be compared to any actual information already stored, the system incorporates this new information. Implicit in this view is the use of meta-meta-rules - rules that governs other rules, instead of rules simply governing the change in knowledge (meta-rules), which allows for a greater degree of autonomy on the system. And finally, the implicit notion of a multi-resolutional hierarchical system, which greatly influenced further works (e.g. Meystel [17]).

\section{Autognomes: Pragmatic Semiotic SYSTEMS}

Based on ideas extracted from the semiotics of Peirce and Morris [1], Eugene Pendergraft developed a system dubbed Autognome [5, 11,12] - or a self-knowing machine. Pendergraft pointed out that the Autognome differs from standard artificial intelligence systems, because it is able to create its own knowledge, using as input the information collected from the environment to which it interacts to. The key concept which allows the working of such machine is the Peircean notion of semiosis, which Pendergraft uses to derive what he calls a pragmatic logic. With this logic, there is paradigmatic shift from an Acquisitive Intelligence, where knowledge is assumed to be "discovered", to a Creative Intelligence, where knowledge is assumed to be "created". Information is stored and manipulated by means of signs. Through semiosis, information is suitable to self-control, self-correction and self-generation. Pendergraft defined the Autognome as a multi-enclave system [5, pp. 148]. The main idea is that the system should be a distinct piece of code enclosed within someone else's code - a recursive appearance of the same code or structure in many different positions, working in multiple levels of actuation (which in some sense looks like Pospelov's notion of a multiresolutional system). According to Pendergraft, there are 3 different types of information transformation processes, which can be roughly associated to: information clustering, associations between clusters and associations between associations. He calls these three processes as the monadic, dyadic and triadic modes of semiosis. According to him, a fully semiotic system can be constructed from components of just three basic types: engineered to carry out these monadic, dyadic and triadic modes of semiosis. The reader should notice that Pendergraft relates these three kinds of components to different kinds of processes - perceptual, conceptual and valuational. This division is based on his interpretation of Peirce's category theory - the foundation of his semiotic system. When assembled together, components of these three types are said to form an enclave capable of performing tri-relative semiosis [5, pp. 135], which Pendergraft defines as the entanglement of abductive, deductive and inductive inferences, structured to create abstract representations for a given set of signals, and use this abstract representation to perform actions on the environment.
So, each enclave is constituted by three components hierarchically connected and responsible for carrying out a specific mode of semiosis. Assuming generically a component responsible for treating the $n$-th mode, we have two main cycles operating on it. The first is the inquiry cycle, which is responsible for collecting information from a previous component (or the real world), and translate this information in terms of a given knowledge. The second cycle is the performance cycle, responsible for using the current stored knowledge to command actions on the component responsible for the (n-1)-th mode. The knowledge in the n-mode component is used as input to the $(n+1)$-mode component and is also affected by commands from this component.

A working version of the Autognome is currently being commercialized by $\mathrm{Ai}^{2}$, a company in U.S., and have been used in many different kinds of applications, like written text automatic classification, intelligent medical instruments, forecast and advice for energy comsumption optimization and knowledge management collaborative tools. In theory, the Autognome can be used in any kind of system that collects data from a given environment, and want to structure and abstract this data, and use it to perform some kind of action. The enclave-type structure of the Autognome allows it to be integrated to any kind of software system where automatic data classification and abstraction is an issue.

\section{The Multi-Resolutional Semiosis}

In the beginning of the 90's, James Albus published a seminal paper proposing a general architecture for any kind of intelligent system [13]. According to Albus, an intelligent system is composed of 4 basic modules $[13,14,15]$ : sensorial processing, world modeling, value judgment and behavior generation. As a generalization of this architecture, Albus further developed a reference architecture for an intelligent system [16] named RCS (Real-Time-Control System). It was normatized by NIST, being used in many different applications developed at its Intelligent Systems Division. ${ }^{3}$

Using the reference architecture from Albus, Meystel proposed to model the interaction among the modules as a recursive process, which he called a Multiresolutional Semiosis [17]. He developped a very particular model, according to which semiosis is a process involving recursive cycles where sensing, interpretation and actuation do occur in multiple levels of resolution. The kernel of this process is the cyclic application of three main elementary operators: Grouping, Focusing of Attention and Combinatorial Search (GFACS). GFACS occurs within the World Model, which more than a simple repository of information is an active module. According to him, GFACS constitutes "an elemen-

\footnotetext{
${ }^{2} \mathrm{http}: / /$ www.asitisinc.com

${ }^{3}$ http://www.isd.mel.nist.gov
} 
tary unit of intelligence" which, being used in multiple levels of resolution, will build the intelligent behavior of a system.

The Albus-Meystel approach was used to build many different kinds of intelligent systems, most of them robotic systems. Among them a "baby robot" that learns from its interaction with the environment, controllers for unmanned ground vehicles, submarines and other kinds of military applications.

\section{OTHER APPROACHES}

\section{Organizational Semiotics}

The basic idea of Organizational Semiotics is to model organizations (biochemical structures, business organizations, societies, etc.) from the point of view of sign processing $[18,19]$. The studies of Kecheng Liu [18], cover the aspects of business organizations, where people exert a decisive influence on the organizational behavior, by means of individual or group sign exchange. The main framework, according to Liu, is the MEASUR (Methods for Eliciting, Analyzing and Specifying User's Requirements), a set of norm-oriented methods for business modeling and software requisites specification, comprising five great methods for business modeling: Problem Articulation, Semantic Analysis, Norm Analysis, Control and Communication Analysis and Meta-Systemic Analysis. The main contribution of Liu is to use semiotics for the development of more efficient procedures to generate standard software systems (software engineering techniques). Other approaches on this same field are those from [20], and their CASTOR: (Conversion of knowledge And Semiotic Theories of Organization and Representation) Project, and those from [21,22], on the relation between organizations, multi-agent systems and semiotics.

\section{SCIPS - The Semiotic Cognitive Information Processing}

Semiotic Cognitive Information Processing Systems (SCIPS) [23,24] models the emergence of semiosis as a self-organized process based on combinatorial and selective language universal constraints. Rieger recognizes two kinds of inputs in cognitive systems: immediate inputs, and mediate inputs. Immediate inputs are direct phenomena observed in an environment by a cognitive system. Mediate inputs are those enabled by texts. For mediate inputs to acquire meaning, Rieger proposes a meaning constitution procedure, based on "Language Games" performed by different cognitive systems, using the environment for communication [23]. His proposal is to apply text analysis algorithms to operate in different levels, measuring the syntagmatic and paradigmatic relations in text fragments exchanged by SCIPS actuating under a same environment [25].

\section{The Semiotic Agents}

Luís Rocha and Cliff Joslyn developped an approach strongly inspired on biological complex systems, and based on the notion of semantic closure, as originally stated by Pattee [26]. Joslyn [27] introduces the idea of semantic closure on the context of system theory and cybernetics. This idea allows to define selection procedures as metasystemic levels required for obtaining semantic relations in control systems. In [28], they perform an analysis of sociotechnical organizations as multi-agent systems, where each agent is viewed as a semiotic entity -- entities which communicate each other by means of the interchange of messages. They discuss several aspects related to the design of semiotic agents in artificial environments [29], such as the requirement of creation of a "virtual physics" ruling the environment, in order to allow the phenomenon of selforganization to take place, the establishment of generalized control relations between the agents and the development of local communication mechanisms, in order to share knowledge among a community of agents. The authors emphasis is on the development of modeling strategies for multiagents systems, taking in consideration the phenomenon of behavior emergence, such as in the fields of complex systems and artificial life.

\section{Perlovsky's Intellectual Systems}

Perlovsky [30,31] elaborates on many different aspects where concepts from cognitive sciences and semiotics are used on the development of intelligent control systems. He emphasizes the inseparable integration among three kinds of signs on the processes of perception and cognition - Designative (described by internal models), Appraisive (described by similarity measurings), Prescriptive (described by adaptation laws and other behavioral acts). To develop the mathematical calculus involved in such considerations, he developed his Modeling Field Theory, described in details in [31]. Perlovsky does an analysis of the phenomena of perception and cognition both from the point of view of mathematics and psychology. Perception is related to concept models as subsets of sensorial data collected from the world. Processes involving the manipulation and transformation of signs are so viewed as key-elements to explain the functioning of perception and cognition.

Perlovsky developed a military target search intelligent system (intelligent target tracker), which was implemented in a joint project between U.S. air force and navy. This system is capable of detecting an enemy on a visual field and tracking its movement along the battlefield.

\section{Ecological Semiotic Approach}

The main contribution of Prueitt to computational semiotics is the attempt to renew the actual techniques for knowledge management, using semiotics as a supporting paradigm. In $[32,33]$, Prueitt developed what he considered a set of new methodologies, inspired on the work of Pospelov on applied 
semiotics, for the creation of a new generation of information technology, which he called "The 21th century Knowledge Management", or "Knowledge Science”.

\section{The Semiotic Machines and Knowledge Robots}

Gerd Döben-Henisch introduced the notion of a semiotic machine [34] as a "device able to reconstruct the common structures of human experience in terms of sign processing". In his conception, Semiotic Machines should be used for the representation of the internal processes of a -not necessarily human- sign user, in order to allow its implementation in a computational system. Based on the idea of a semiotic machine, Döben-Henisch derived the notion of a knowledge robot, or knowbot [35] -- a system able to collect knowledge from its environment and use this knowledge in order to decide its own actions. These knowbots are capable of 'learning' their environment at the same time they interact to a human "teacher", which by an appropriate interface, convey symbolic information to be associated to the experiences being perceived by the knowbot. This process, as reported by Döben-Henisch, "tries to mimic the way children use when apprehending their language". In his KIP (Knowbotic Interface Project) [36], a kind of artificial life experiment, he built a tridimensional virtual world where a knowbot is able to move itself autonomously, learning objects from environment, and receiving text strings from a human operator, which it associates to its perceptual information, acquiring a kind of very primitive type of language.

\section{Conclusions}

Our aim here was to briefly present different projects that attempts to put together theoretical semiotics and AI techniques in order to create new kinds of intelligent systems. This interdisciplinary area, based on investigations of sign processes, and dedicated to the development of artificial intelligent systems is dubbed Computational Semiotics. Even though the main developments in the area stayed apart from the mainstream for about 25 years, during the last decade it started to catalyze the attention of a growing number of researchers (see the footnote 1), being the foundation for new kinds of theoretical frameworks and technologies.

The fields of Intelligent Control and Intelligent Systems, despite being studied and developed for more than 40 years, are still far from reproducing completely the same kind of intelligent behavior as it is found in animals and in men. Computational Semiotics proposes a new kind of approach to intelligent control and intelligent systems, where an explicit account for the notion of sign is prominent. This focus on the notion of sign, allows the inheritance of a large body of theory developed under the scope of semiotics studies, that are put into service in order to help artificial intelligence to walk through new frontiers, and bridge theoretical gaps that disturbs artificial intelligence studies since a long time (e.g. the symbol grounding problem[37]).
In this work, we tried to summarize the most representative research projects in Computational Semiotics, collecting a comprehensive set of references in literature, where the reader may start his introduction to the field. Considering the relevance of Computational Semiotics for future developments in Artificial Intelligence, as we envision, we understand that this was the major contribution of this work.

\section{ACKNOWLEDGEMENTS}

R.G. is funded by a grant from CNPq (\#300123/99-0); J.Q. is funded by a grant from FAPESP (\#02/09763-2).

\section{REFERENCES}

[1] Noth, W. - Handbook of Semiotics (Advances in Semiotics) - Indiana University Press, 1995.

[2] Pospelov, D.A., Principles of Situational Control, Engineering Cybernetics, Moscow, 1971(2):216-222.

[3] Pospelov, D.A. - Situational Control : Theory and Practice - unpublished translation from the original in russian, edited by Nauka Publishers, Moscow, 1986.

[4] Pendergraft, E.P. - Self-organizing Linguistic Sytems Linguistic Research Seminar, Santa Monica, California, 1964.

[5] Pendergraft, E.P. The Future's Voice - Intelligence Based on Pragmatic Logic - Technical Report - Creative Intelligence Incorporated, DRAFT (FATE), Jasper, EUA, June 1993.

[6] Pospelov, D.A. and Zhelezov, Zh. Y. - On a Class of Large Systems - Engineering Cybernetics, Moscow, 1970(2):243-246.

[7] Pospelov,D.A. and Yeimov, Ye. I., Semiotic Models in Planning Problems of Artificial Intellect Systems, Engineering Cybernetics, Moscow, 1977(5):37-43.

[8] Osipov, G.S., Origins of Applied Semiotics, ECAI 2000, 14th European Conference on Artificial Intelligence, 2000.

[9] Sulosky, M.F., Semiotics Situational Control - JSMtype Reasoning and Q-analysis, Intelligent Systems: a semiotic perspective, Proceedings of the 1996 International Multidisciplinary Conference, Vol. II: Applied Semiotics, Gaithersburg, Maryland, 1996.

[10] Waterman, D.A. - A Guide to Expert Systems - Addison Wesley Publishing Company, 1986.

[11] Autognomics, Corp., White paper, A Skeleton Autognome, Autognomics Corp., EUA, 1997.

[12] Autognomics, Corp., Autognomics Technology - Brief Overview of Autognomics, Autognomics Corp., EUA, , 2001. 
[13]Albus, J.S. - Outline for a Theory of Intelligence IEEE Transactions on System, Man and Cybernetics, vol. 21, n. 3, May/June 1991.

[14]Albus, J.S.; Meystel, A.M. - Engineering of Mind: An Introduction to the Science of Intelligent Systems, John Wiley \& Sons; ISBN: 0471438545, 2001.

[15] Meystel, A.M.; Albus, J.S. Intelligent Systems: Architecture, Design, Control - Wiley-Interscience; ISBN: 0471193747, 2001.

[16]Albus, J. S. - The NIST Real-Time Control System (RCS): An Approach To Intelligent System Research, J.Expt.Theor.Artif.Intell - 1997 - pp.157 -162.

[17]Meystel, A.M. - Semiotic Modeling and Situation Analysis : An Introduction - AdRem Inc. - Bala Cynwyd, PA, USA, 1995.

[18]Liu, K. Semiotics in Information Systems Engineering - Cambridge University Press, ISBN: 0521593352, 2000.

[19]Liu, K.; Clarke, R.J.; Andersen, P.B.; Stamper, R.K. 2001) Information, Organisation and Technology: Studies in Organisational Semiotics - Kluwer Academic Publishers, ISBN: 0792372581, 2001.

[20] Van Heusden, B.; Jorna, R.J. - "Reconsidering the Standard: A Semiotic Model of Organisations" In: Kecheng Liu, R.J. Clarke, P.B. Andersen \& R.K. Stamper (Eds.) Coordination and communication using signs: Studies in Organisational Semiotics 2. Dordrecht: Kluwer Academic Publishers, p. 153-67.

[21] Gazendam, Henk W.M. (2001). "Semiotics, Virtual Organizations, and Information Systems ", in: Kecheng Liu, Rodney J. Clarke, Peter Bøgh Andersen and Ronald K. Stamper (eds.). Information, Organisation and Technology: Studies in Organisational Semiotics. Boston: Kluwer Academic Publishers, 2001: 1-48.

[22] Gazendam, Henk W.M., and René J. Jorna. (1998). "Semiotics, Multi-Agent Systems and Organizations.." Proceedings of the Joint Conference on the Science and Technology of Intelligent Systems, September 14-17, 1998, Gaithersburg, MD, USA. Piscataway, NJ: IEEE: 84-89. ISBN 0-7803-4423-3.

[23]Rieger, B.B. - Situations, Language Games, and SCIPS. Modeling semiotic cognitive information processing systems - in: Albus, J./ Meystel, A./ Pospelov, D./ Reader, T. (eds.): Architectures for Semiotic Modeling and Situation Analysis in Large Complex Systems (Proceedings of The 1995 ISIC-Workshop, Monterey: the 10th International IEEE-Symposium on Intelligent Control), Bala Cynwyd, PA. (AdRem Inc.) 1995, pp. 130-138

[24]Rieger, B.B. - Semiotics and Computational Linguistics. On Semiotic Cognitive Information Processing - in: Zadeh, Lotfi A. / Kacprzyk, Janusz (eds.): Computing with Words in Information/ Intelligent Systems I. Foundations
[Studies in Fuzziness and Soft Computing 33], Heidelberg, (Physica Verlag) 1999, pp. 93 - 118

[25]Rieger, B.B. - A Systems Theoretical View on Computational Semiotics. Modeling text understanding as meaning constitution by SCIPS, in: Proceedings of the Joint IEEE Conference on the Science and Technology of Intelligent Systems (ISIC/CIRA/ISAS-98), Piscataway, NJ (IEEE/Omnipress) 1998, pp. 840-845

[26]Pattee, H.H. - Cell psychology: An evolutionary approach to the symbol-matter problem - Cognition and Brain Theory 5 (4), 325-341, 1982.

[27]Joslyn, C. The Semiotics Of Control And Modeling Relations In Complex Systems - BioSystems 60 (2001) 131-148.

[28]Joslyn, C.; Rocha, L.M. - Towards Semiotic AgentBased Models of Socio-Technical Organizations - AI, Simulation and Planning (AIS 2000) Conference, Tucson, Arizona, USA, 2000.

[29] Rocha. L. M.- Models of embodied, evolving, semiosis in artificial environments. In: Landauer, C., Bellman, K.L. Proceeding of the virtual world and simulation conference. The society for Computer Simulation, pp 233-238, 1998

[30] Perlovsky, L. - Semiotics, Mind and Architecture of Target Tracker - Proceedings of the IEEE ISIC/CIRA/ISAS Joint Conference -Gaithersburg, MD. Sep 1998.

[31] Perlovsky, L. - Neural Networks And Intellect - Oxford Press - ISBN 0195111621, 2000.

[32] Prueitt, P.S., Is Computation Something New?, Intelligent Systems: a semiotic perspective, Proceedings of the 1996 International Multidisciplinary Conference, Vol. II: Applied Semiotics, Gaithersburg, Maryland, 1996.

[33] Prueitt, P. - Foundations of Knowledge Management for the 21st Century, BCN Group Inc., EUA, 1999.

[34]Döben-Henisch, G. - Semiotic Machines - Theory, Implementation, Semiotic Relevance - 8th International Semiotic Congress of the German and the Netherlands Semiotic Societies - Amsterdam, August 1996.

[35]Döben-Henisch, G. Erasmus, L., Hasebrook J. - Knowledge Robots for Knowledge Workers: Self-Learning Agents connecting Information and Skills, in: Intelligent Agents and Their Applications (Studies in Fuzziness and Soft Computing, Vol. 98), L. C. Jain, Zhengxin Chen, Nikhil Ichalkaranje (eds.), Springer, New York, 2002, pp.59-79

[36]Döben-Henisch, G.; Klöckner, M. - Agents with Consciousness - Knowbotic-Interface Project - KIP - Technical Report - INM - Institut für Neue Medien - Daimlerstr. 32 Frankfurt, Germany - August 1995. 